\title{
Papillary thyroid carcinoma micro-deposits in cervical lymph nodes without intra-thyroid malignancy: A comment in consideration of the latest American Thyroid Association guidelines
}

\author{
EVANGELOS KARVOUNIS $^{1}$, ARISTOTELIS KECHAGIAS ${ }^{2}$, IOANNIS KAPPAS ${ }^{1}$, \\ CHRISTINA IOAKIMIDOU $^{3}$ and THEODOROS FILLIPIDIS ${ }^{3}$ \\ ${ }^{1}$ Department of Endocrine Surgery, Center of Excellence, Euroclinic Hospital, Athens 115221, Greece; \\ ${ }^{2}$ Department of Digestive and Endocrine Surgery, Kanta-Häme Central Hospital, Hämeenlinna 13530, Finland; \\ ${ }^{3}$ Department of Pathology, 'Micromedica' Histopathological Laboratory, Athens 16344, Greece
}

Received July 10, 2020; Accepted April 4, 2021

DOI: $10.3892 / \mathrm{mco} .2021 .2326$

\begin{abstract}
Differentiated thyroid cancer (DTC) represents the vast majority of all thyroid cancers, with the papillary variant being the most common. According to the previous 2009 American Thyroid Association (ATA) guidelines, papillary thyroid microcarcinoma (PTMC; $\leq 1 \mathrm{~cm}$ in diameter) exhibiting cervical lymph node metastasis corresponded to an intermediate-risk group for recurrence or metastasis. However, the latest 2015 ATA guidelines advocate that a patient with PTMC is low-risk if there are $\leq 5$ regional node micrometastases. This means that therapeutic radioactive iodine (RAI) is not required. The current study reports a rare case of a patient who underwent total thyroidectomy due to multi-nodular goiter where the pathologic specimen exhibited two PTMC foci in regional lymph nodes, but no primary cancer was identified in the thyroid despite thorough examination of the thyroid parenchyma. The etiology of such results is unknown and it was hypothesized that it may be the consequence of insufficient pathologic examination or due to the regression of a primary PTMC in the thyroid. Moreover, the risk-stratification of cases with intra-lymph node PTMC without any evidence of primary cancer in the thyroid is not considered in the
\end{abstract}

Correspondence to: Dr Aristotelis Kechagias, Department of Digestive and Endocrine Surgery, Kanta-Häme Central Hospital, 20 Ahvenistontie, Hämeenlinna 13530, Finland

E-mail: aristotelis.kechagias@yahoo.gr

Abbreviations: DTC, differentiated thyroid cancer; PTC, papillary thyroid carcinoma; PTMC, papillary thyroid microcarcinoma; ATA, American Thyroid Association; RAI, radioactive iodine

Key words: differentiated thyroid cancer, papillary thyroid carcinoma, papillary thyroid microcarcinoma, American Thyroid Association, cervical lymphnode metastases, microfoci, micrometastases
ATA recommendations. The aim of the current report was to elucidate the risk-stratification of this rare occurrence and to reconsider the possible etiologies. By extrapolating the latest ATA recommendations concerning a patient with a known primary PTMC and $\leq 5$ metastatic micro-foci (thus the only difference between cases being the absence of a primary tumor), it was concluded that the patient should be considered low-risk. As a consequence, RAI therapy should be deemed as unnecessary despite the presence of lymph node microfoci. Moreover, it was proposed that cervical lymph node PTMC with no evidence of a primary tumor in the thyroid could be the consequence of normal thyroid tissue micro-deposit progression to cancer within the lymph node, which is a rare benign entity.

\section{Introduction}

Differentiated thyroid cancer (DTC) represents the vast majority (90\%) of all thyroid cancers, and it includes papillary and follicular neoplasms (1). According to epidemiologic estimations there were 63.000 new cases of DTC in the United States in 2014 compared to 37.200 in 2009 (1). This trend has been attributed almost entirely to the increase of the incidence of the papillary thyroid carcinoma (PTC) which is the most common form of DTC (1). In particular, the increasing use of neck ultrasonography resulted in a considerable rise of the new cases of PTC $\leq 1 \mathrm{~cm}$ which is distinguished as the papillary thyroid microcarcinoma (PTMC) $(1,2)$. Additionally, there has been a doubling of the clinically-occult thyroid cancers which are detected incidentally during the pathologic exam after thyroidectomy for benign indication (2).

PTC involves cervical lymph node metastases in $20-50 \%$ of the patients, and the frequency of lymph node micrometastases ( $<2 \mathrm{~mm}$ ) may approach $90 \%$ in some series (1). In addition, there have been interesting reports of rare cases of incidental detection of PTMC in cervical lymph nodes without identifiable tumour within the thyroid gland (3). These extra-thyroid PTMC foci have been stipulated as metastases from an unknown 
primary deriving from the thyroid parenchyma despite thorough pathologic examination of the specimen (3). Due to their rare occurrence, their clinical potential is not discussed in the American Thyroid Association (ATA) guidelines (1).

With this communication we aim to provide an expert opinion concerning the clinical importance of PTC microscopic foci in cervical lymph nodes with no identifiable tumour within the thyroid, despite meticulous pathologic examination of the gland. For this objective, a clinical case is briefly described and it is discussed in view of the revised recommendation of the available ATA guidelines (1) regarding a present primary PTMC with lymph node micrometastases.

\section{Case report}

A 43-year old white male patient was referred to the Department of Endocrine Surgery, Euroclinic Hospital, Athens, Greece due to thyroid multinodular goiter in September 2017. He had a feeling of mild pressure in the neck in the past few months, which was exhibited particularly during swallowing. The largest nodule was $29 \mathrm{~mm}$ and displayed TI-RADS 2 characteristics in imaging. According to his anamnesis the nodule had grown in dimension. Thyroglobulin, calcitonin and CEA levels were normal and the patient was euthyroid. Further observation or surgical options were discussed with the patient and a total en-block thyroidectomy was performed in consideration of his preferences.

The surgical specimen of total thyroidectomy was inserted in buffered formalin (1:10) and the entire gland underwent thorough macroscopic and histopathologic examination by two experienced endocrine pathologists. The thyroid gland weighted $22 \mathrm{~g}$. The dimensions of the right lobe were $4.5 \times 3 \times 1.7 \mathrm{~cm}$, and the dimensions of the left lobe were $5 \times 2.5 \times 2.2 \mathrm{~cm}$ with the inclusion of the isthmus and the pyramidal lobe. The specimen capsule was intact. The thyroid gland was seriously sectioned and sampled into its entirety with a total of 26 parafine blocks and a mean width of $2.1 \mathrm{~mm}$ for each slice. Hematoxylin and eosin staining were employed for morphologic assessment. Commercially available immune antibodies were used to detect thyroglobulin receptors (Thermo, 1:600, Ab-6 coctail). Pre-digestion was performed for $3 \mathrm{~min}$ with $0.05 \%$ protease VIII in a $0.1 \mathrm{M}$ phosphate buffer with $\mathrm{ph}=7.8$ at $37^{\circ} \mathrm{C}$. The findings were consistent with thyroid multinodular hyperplasia and there was no detectable tumor in the thyroid. Two of the excised lymph nodes from the central neck compartment displayed multiple confluent papillary micro-formations (0.3-1 $\mathrm{mm}$ of diameter) with clear irregular nuclei and papillary thyroid cancer phenotype. In immunohistochemistry the carcinoma cells were positive for thyroglobulin receptors. The microformations are shown in Fig. 1 with positive thyroglobulin immunostaining.

The existing guidelines did not consider at all PTMC cases with an absent primary. The decision of the multi-disciplinary neck-endocrine oncological meeting classified the patient in the group of intermediate risk for recurrence or metastasis. The patient underwent post-operative radioactive iodine (RAI) therapy and a standard treatment-policy was employed in the course of this study. At three years and three months of follow-up the is no sign of recurrence and the serum thyroglobulin level remains low $(<0.3 \mathrm{ng} / \mathrm{ml})$.
The patient consented in written for the publication of his case in the form of a case report. Approval from the ethical committee is not required in our hospital for case report publications.

\section{Discussion}

The presence of PTC tissue in cervical lymph nodes without identification of any cancer in the thyroid itself is a very uncommon event with unknown etiology. So far, it has been considered to represent lymph node metastatic disease from an occult primary thyroid microcarcinoma (3). A recent review of the literature by $\mathrm{Xu}$ et al has reported that there have been only 9 well-documented cases of metastatic thyroid carcinoma in cervical lymph nodes with no detectable primary, despite a thorough histopathologic examination of the thyroid (3).

The meticulous pathologic examination that was performed in the case that we describe herein failed to show any original cancer in the thyroid parenchyma. The mechanism of this rare phenomenon remains unknown (3) as the attainment of direct proof is more than challenging. Therefore, possible suggestions concerning its etiology are rather theoretic. It has been proposed that even exhaustive histopathologic examination may miss a microcarcinoma $<3 \mathrm{~mm}$ in size, which is a lesion that may have the potential to develop metastases $(3,4)$. In our case, the histologic sections had a mean thickness of $2.1 \mathrm{~mm}$ which provided a good possibility for the detection of even smaller lesions (less than 1-2 mm). The second theory suggests the probability that the primary tumor regresses to fibrotic tissue within the thyroid parenchyma (3). Bearing in mind the comprehensive pathologic examination in our case-which included an extended re-evaluation of the specimen -, the undersigned authors consider and propose an additional alternative etiologic possibility without rejecting the existing ones. A PTMC focus in the thyroid parenchyma may be indeed absent in some of the cases. The explanation could be hidden in the uncommon but well-documented (5) presence of inclusions of benign thyroid tissue in cervical lymph nodes. Consequently, the possibility that such benign deposits evolved to intra-lymph node PTC micro-deposits due to the action of unknown biochemical or molecular stimuli can not be dismissed.

Concerning the clinical aspect of our case, it should be highlighted that the current guidelines do not deal at all with the rare entity of PTC micro-foci in the cervical lymph nodes with no detectable thyroid primary (1). Proper disease risk-stratification should be the main objective also in cases of PTC micro-inclusions in cervical lymph nodes with an occult or questionable primary tumor in order to guide therapy and follow-up. As the ATA guidelines consider only the eventuality of a known primary PTC with or without nodal metastases (1), we believe that the closest extrapolation that can be dragged for our case is to assume the same lymph node invasion pattern in a patient with a detected PTMC. The previous version (2009) of the ATA guidelines advocated that patients with a primary PTMC and any cervical lymph node metastasis belong to the intermediate-risk group for recurrence or metastasis (6). However, the subsequent version of these guidelines which was published in 2015 considered that the clinical implication of regional lymph node micrometastases may be less significant 

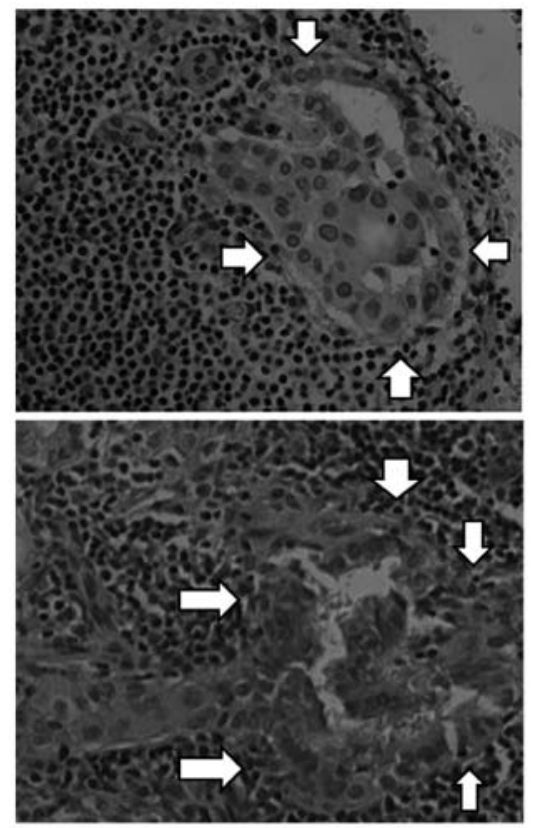

Figure 1. Microscopic image of papillary thyroid microcarcinoma inclusion in two regional lymph nodes (arrows) after total thyroidectomy for multi-nodular goiter (magnification x200). Thyroglobulin immunostaining was conducted.

than previously thought (1). The new ATA proposal was therefore modified accordingly and downgraded the patients with a known primary PTMC and $\leq 5$ of regional lymph node micrometastases in the low-risk group (1).

The herein case with two local micro-foci of PTC in regional lymph nodes and absent or occult intra-thyroid cancer (despite thorough pathologic examination) would correspond rather to the intermediate-risk group of the 2009 ATA risk stratification (6). However, by extrapolating the information provided in the latest ATA guidelines (1) for patients with a known primary PTMC and $\leq 5$ regional node micrometastases, our case displays a pattern with better association to a weak malignant potential and therefore it should be included in the low-risk group. This change in the stratification of the risk of the same patient between the current and the previous version of the ATA recommendations is clinically important as both versions advocate that RAI adjuvant therapy should be administered in the intermediate-risk patients, but not for those with low-risk. Low-risk patients that have undergone total thyroidectomy should have routine post-operative measurements of serum thyroglobulin with the consideration of ultrasound and/or RAI scanning for diagnostic purposes (1). In turn, a different case with multiple (>5) PTC micro-inclusions in lymph nodes and without an identifiable primary after total thyroidectomy would better pertain to the intermediate-risk and therefore adjuvant RAI should be considered.

It should be noted that our specimen was not tested for a BRAFV600E mutation, however in our opinion this poses only a marginal limitation to this study. The current ATA recommendations are not routinely recommending BRAF mutational evaluation for initial postoperative risk stratification in DTC (1). In particular, PTMC harboring BRAFV600E mutations with no other worrisome features are considered as low-risk lesions (1). It could be possible that a BRAFV600E mutation is followed by increased risk of recurrence or mortality in larger DTCs, however there is not enough evidence yet that this factor could be used in isolation for the risk-stratification (1). Moreover, the follow-up of our case reached 3,25 years. A concern could be raised if this patient would continue to remain disease-free or not, as the possibility for a recurrence is not well documented in literature for a PTMC after the course of 5 years $(1,7)$. However, it is known that the initial risk estimates are modified during time for DTC and they improve if there is no evidence of disease during follow-up $(1,7)$. It should be noted that the risk of recurrence in patients initially classified as at intermediate risk drops from 35 to $1-2 \%$ if they display excellent response at 18 months of follow-up (1,7). Bearing in mind this example and the excellent course of our (low-risk) patient during follow-up it could be inferred that the probability of a recurrence could be even less than $1 \%$. A very interesting review by $\mathrm{Xu}$ et al identified five well-documented cases of metastatic PTMC without evidence of any thyroid primary (3). Half of these patients were positive for BRAFV600E, two patients received postoperative radioiodine therapy (with 2 and 5 positive lymph nodes, respectively), and all five were alive and without evidence of recurrence at a median follow-up of 2.2 years (3). This additional evidence concurs that PTMC foci in lymph nodes with absent primary is rather a low-risk entity. In conclusion, patients with a few ( $\leq 5)$ PTC microscopic ( $<2 \mathrm{~mm}$ ) deposits in cervical lymph nodes with an occult (or questionable) primary tumor appear to bear a low potential for recurrence or metastasis after total thyroidectomy. Extrapolating from the latest ATA guidelines concerning the same lymph node-involvement pattern but a detected primary PTMC in the pathologic examination, adjuvant RAI therapy should be regarded rather as an overtreatment and should be avoided.

\section{Acknowledgements}

Not applicable.

\section{Funding}

No funding was received.

\section{Availability of data and materials}

Data sharing is not applicable to this article, as no datasets were generated or analyzed during the current study.

\section{Authors' contributions}

EK and AK conceived the current study. EK, AK, IK, CI and TF designed the current study. EK, AK, IK, CI and TF acquired the data. EK, AK, IK, CI and TF drafted the manuscript. EK, $\mathrm{AK}$ and $\mathrm{CI}$ revised the manuscript. $\mathrm{EK}$ and $\mathrm{AK}$ provided final approval for the manuscript. All authors read and approved the final manuscript. EK and AK confirm the authenticity of all the raw data.

\section{Ethics approval and consent to participate}

Not applicable. 


\section{Patient consent for publication}

The patient provided written informed consent for the publication of his data.

\section{Competing interests}

The authors declare that they have no competing interests.

\section{References}

1. Haugen BR, Alexander EK, Bible KC, Doherty GM, Mandel SJ, Nikiforov YE, Pacini F, Randolph GW, Sawka AM, Schlumberger M, et al: 2015 American thyroid association management guidelines for adult patients with thyroid nodules and differentiated thyroid cancer: The American thyroid association guidelines task force on thyroid nodules and differentiated thyroid Cancer. Thyroid 26: 1-133, 2016.

2. Siegel R, Ma J, Zou Z and Jemal A: Cancer statistics 2014. CA Cancer J Clin 64: 9-29, 2014
3. Xu B, Scognamiglio T, Cohen PR, Prasad ML, Hasanovic A, Tuttle RM, Katabi N and Ghossein RA: Metastatic thyroid carcinoma without identifiable primary tumor within the thyroid gland: A retrospective study of a rare phenomenon. Hum Pathol 65: 133-139, 2017.

4. Ghossein R, Ganly I, Biagini A, Robenshtok E, Rivera M and Tuttle RM: Prognostic factors in papillary microcarcinoma with emphasis on histologic subtyping: A clinicopathologic study of 148 cases. Thyroid 24: 245-253, 2014.

5. León X, Sancho FJ, García J, Sañudo JR, Orús C and Quer M: Incidence and significance of clinically unsuspected thyroid tissue in lymph nodes found during neck dissection in head and neck carcinoma patients. Laryngoscope 115: 470-474, 2005.

6. American Thyroid Association (ATA) Guidelines Taskforce on Thyroid Nodules and Differentiated Thyroid Cancer; Cooper DS, Doherty GM, Haugen BR, Kloos RT, Lee SL, Mandel SJ, Mazzaferri EL, McIver B, Pacini F, et al: Revised American thyroid association management guidelines for patients with thyroid nodules and differentiated thyroid cancer. Thyroid 19: $1167-1214,2009$.

7. Lamartina L and Handkiewicz-Junak D: Follow-up of low risk thyroid cancer patients: Can we stop follow-up after 5 years of complete remission? Eur J Endocrinol 182: D1-D16, 2020. 